\title{
Ein Analogrechenverfahren zur Analyse biologischer Anpassungsvorgänge mit der Methode der Thermodynamik irreversibler Prozesse
}

\author{
EKKEHARD ZERBST \\ Pbysiologisches Institut der Freien Universität Berlin
}

\begin{abstract}
An analogue computing system for analysis of biological adaptation processes using the method of the thermodynamics of irreversible processes. By means of theoretical considerations concerning functional biological adaptations, attempts have been made (1) to explain these adaptations on the basis of transitional flux equilibria of cellular metabolic energy processes, (2) to obtain a comprehensive description using transport equations of irreversible thermodynamics, and (3) to analyse the adaptations on the basis of metabolic feedback system. An analogue computer was used for mathematical calculations. The model of a biological reaction train was set up using a 4-pol system with series of coupled $\mathrm{RC}$-members. Substrate concentrations or driving forces were represented as potentials, velocity constants as conductances or reciprocal time constants, and substrate- or energy fluxes as electric currents. Taking into consideration metabolic feed-back systems, it has been shown that all five types of the temperature-capacity adaptation can be explained on the basis of energetic steady state transitions and may be analysed qualitatively and quantitatively using thermodynamics of irreversible processes. Based on experimental calculations with this model, specific biological investigations of the temperature-capacity adaptation of frog heart rate were untertaken. The experimental results obtained agree well with the theoretical predictions from the model, proving the fundamental value of the application of analogue computer models for solving biological problems. Finally, the possibilities of such elementary energetic cell models, if used as receptor- or neuronmodels in the field of neurophysiology, are discussed, and an example is given. Together with methods of the thermodynamics of irreversible processes, analogue models have also proven useful for solving problems of the biological information theory.
\end{abstract}

\section{EINLEITUNG}

Unter der Einwirkung zeitlich rechteckig verlaufender Milieu-, Licht-, Temperatur- oder Reizveränderungen zeigen Einzelzellen, isolierte Gewebe und Poikilotherme charakteristische Anpassungskurven der biologischen Leistungen. Weil diese Kurven bei unterschiedlichen Objekten und Einwirkungen stets einen prinzipiell gleichen Verlauf haben, ergeben sich bei der Untersuchung biologischer Aupassungsvorgänge drei grundsätzliche Fragen:

1. Ist eine funktionelle Anpassung bereits aus den Grundeigenschaften der Stoffwechselkinetik, d. h. aus Fließgleichgewichtsveränderungen zu erklären? Pütter (1918-1920), Hecht (1931), v. Bertalanffy (1951, 1953), Burton (1939), Denbigh (1948), Hearon (1952), Ranke (1961), Grainger (1959) sowie Behmann \& Merssner (1962) haben diese Frage in unterschiedlichen Ansätzen gestellt. 


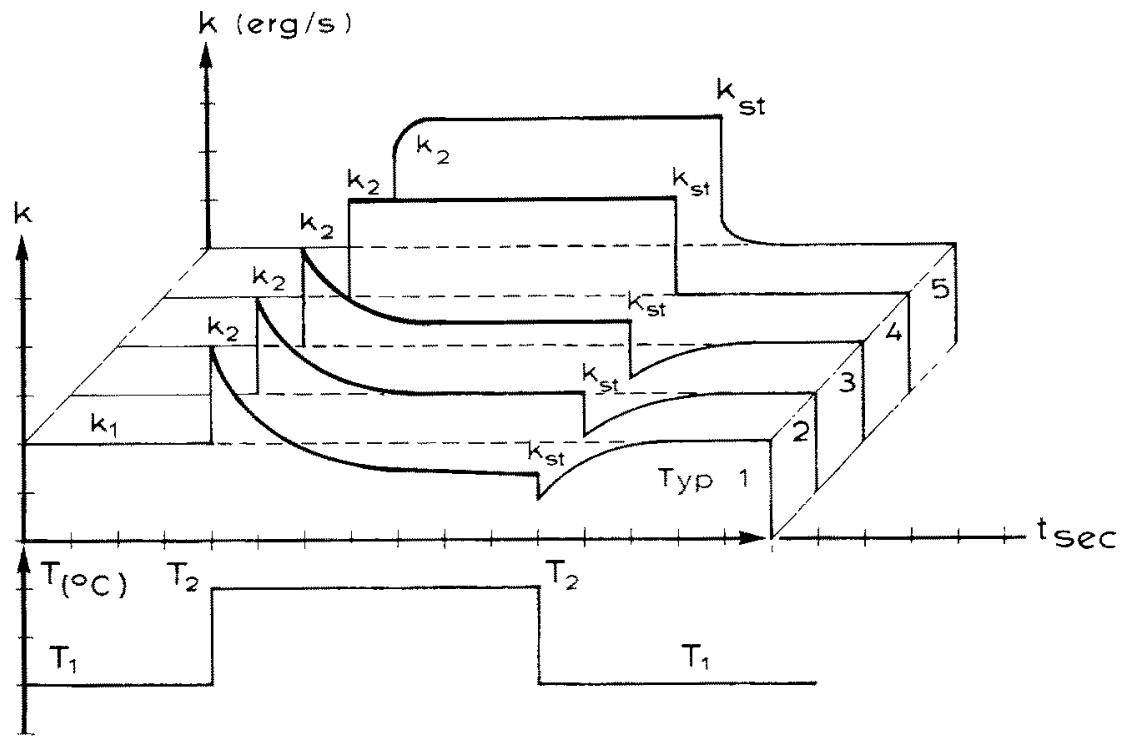

Abb. 1: Zeitlicher Verlauf der Einstellvorgänge bei der Temperatur-Leistungsadaptation. Leistung (k) als Funktion der Temperatur $(\mathrm{T})$ und der Zeit $(\mathrm{t}) . \mathrm{T}_{2}-\mathrm{T}_{1}=10^{\circ} \mathrm{C} ; \mathrm{Q}_{10}=2$. Bei plötzlicher Temperatursteigerung erfolgt eine initiale Leistungszunahme von $\mathrm{k}_{1}$ auf $\mathrm{k}_{2}$. Bei anschließend konstant bleibender Temperatur $\mathrm{T}_{2}$ wird der Leistungs-steady-state $\mathrm{k}_{\mathrm{st}}$ je nach Adaptationstyp eingestellt. Bei Temperatursenkung ergeben sich inverse Züge. (Abszissen- und Ordinatenwerte in willkürlicher Einteilung. Nachzeichnung von Originalregistrierungen eines Modellversuches)

2. Ist die Beschreibung solcher Anpassungsvorgänge mit Transportgleichungssystemen der Thermodynamik irreversibler Prozesse (v. Bertalanffy 1951, 1953, Meixner \& Reik 1959, u. a.) möglich?

3. Lassen sich Analysen der Anpassungsregulationen unter Berüdssichtigung biochemischer Regulationen (Krebs 1956, Hess \& Chance 1959, Holzer 1962) durchführen?

Unsere theoretischen Untersuchungen dieser Frage werden im folgenden am Beispiel der Temperatur-Leistungsanpassung (PRECHT et al. 1955) poikilothermer Objekte diskutiert. Bei diesen Vorgängen treten alle theoretisch nur möglichen Adaptationstypen auf. Die Abbildung 1 gibt schematisch den Anpassungsgang der fünf Typen wieder. Als Leistung wird dabei jede aktive Lebensäußerung thermischer, chemischer, mechanischer und elektrischer Art verstanden. Der Begriff Adaptation besagt nichts weiter, als daß die Zeit als Parameter auftritt.

\section{ERGEBNISSE UND DISKUSSION}

Die Untersuchungen gehen von folgenden 7 Voraussetzungen aus: 1 . Thermodynamisch offene lebende Systeme werden permanent von Stoff- und Energieflïssen durchzogen. 2. Die Funktionsgröße der Systeme ist pauschal der energetischen Umsatzrate proportional zu setzen. 3. Diese Umsatzgeschwindigkeit wird durch Triebkräfte 
$\left(\mathrm{X}_{\mathrm{i}}\right)$ physikalisch-chemischer Potentialgradienten und die entsprechenden Geschwindigkeitskoeffizienten $\left(\mathrm{L}_{\mathrm{ik}}\right)$ bestimmt. 4. Dabei wird jeder energetische Fluß $\left(\mathrm{J}_{\mathrm{i}}\right)$ gemäß den Onsagerschen Reziprozitätsbeziehungen mit allen Triebkräften und Koeffizienten gekoppelt. Das kommt in dem allgemeinen Transportgleichungssystem (1) der Thermodynamik irreversibler Prozesse zum Ausdruck:

$$
\mathrm{J}_{\mathrm{i}}=\sum_{\mathrm{k}=1}^{\mathrm{n}} \mathrm{L}_{\mathrm{ik}} \mathrm{X}_{\mathrm{k}} \quad(\mathrm{i}=1,2,3, \ldots \ldots \mathrm{n})
$$

5. Die biochemischen Triebkräfte werden als homoiostatisch geregelt und konstant bleibend angesetzt. 6. Aus der Gleichung (1) ergibt sich die Form (2)

$$
\mathrm{T}_{\mathrm{ahs} .} \cdot \mathrm{s}=\sum_{\mathrm{i}}^{1} \mathrm{~J}_{\mathrm{i}} \mathrm{X}_{\mathrm{i}}
$$

Danach hat die Entropieerzeugung des offenen Systems multipliziert mit der absoluten Temperatur die Dimension der Leistung. Ihr wird die je Zeit- und Volumeneinheit in bezug auf die Arbeitsleistung des Systems gebundene Energie bzw. Dissipation propor-
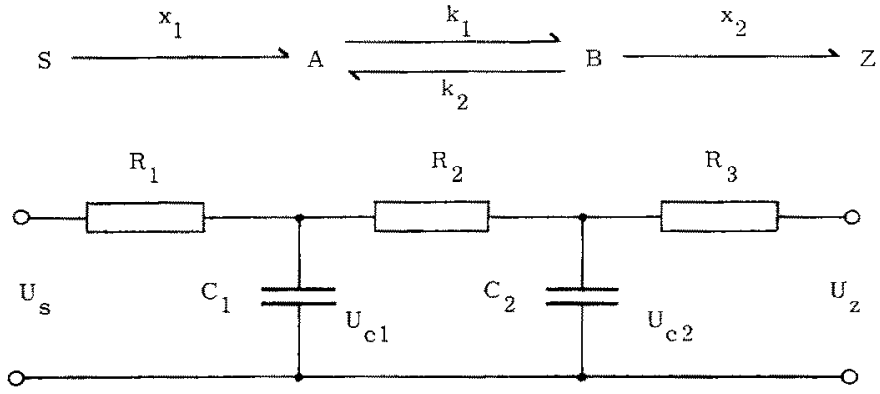

Differentialgleichungen des chemischen und elektrischen vorganges:

$$
\begin{aligned}
& \frac{d c_{a}}{d t}=x_{1}\left(c_{s}-C_{a}\right)-k_{1} c_{a}+k_{2} c_{b} \text { (ehem.) } \\
& \frac{d U c 1}{d t}=\frac{1}{R_{1} C_{1}}\left(U_{s}-U_{c 1}\right)-\frac{1}{R_{2} C_{1}} U_{c 1}+\frac{1}{R_{2} C_{2}} U_{c 2} \text { (elektr.) }
\end{aligned}
$$

ANALOGIEBEZIEHUNGEN:

$$
\begin{aligned}
& \text { Konzentrationen } \\
& \text { Potentiale } \\
& S A B Z \\
& \mathrm{U}_{\mathrm{s}} \mathrm{U}_{\mathrm{c} 1} \mathrm{U}_{\mathrm{c} 2} \mathrm{U}_{z} \\
& \text { Koeffizienten } \\
& \begin{array}{lll}
x_{1} & k_{1} & x_{2}
\end{array} \\
& \text { rezipr. Zeitkonstanten } \\
& \text { bzw. Leitwerte } \\
& \frac{1}{R_{1} C_{1}} ; \frac{1}{R_{2} C_{1}} ; \frac{1}{R_{2} C_{2}} \\
& 1 / R_{1} \quad 1 / R_{2} \quad 1 / R_{3}
\end{aligned}
$$

Abb. 2: Chemischer Reaktionszug im thermodynamisch offenen System und sein elektrisches Ersatzschaltbild 
tional gesetzt. 7. Für Leistungsänderungen des Systems sind demnach Veränderungen der Geschwindigkeitskoeffizienten $\left(\mathrm{L}_{\mathrm{ik}}\right)$ maßgebend. Letztere sind durch Temperaturoder Reizwirkung auszulösen.

Die Fließgleichgewichtsveränderungen energetischer Flüsse und der ihnen proportional gesetzten Leistungsgrößen sind an geeigneten Modellvorgängen mathematisch zu definieren. So haben v. Bertalanffy (1951, 1953), Burton (1939) und Ranke (1962) dem lebenden System einen einfachen, nettomäßig einsinnig verlaufenden Reaktionszug analog gesetzt. Die rein rechnerische Behandlung eines solchen Modelles wird jedoch bei speziellen Fließgleichgewichtsübergängen relativ schwierig und unanschaulich, besonders dann, wenn den primären Regulationen über einfache Fließgleichgewichtsveränderungen noch sekundäre Regulationen nach Art von Rückkoppelungsvorgängen parallel laufen (Definition der Regulationen nach v. BERTALANFFY (1951, 1953). Wir haben deshalb das in Abbildung 2 dargestellte elektrische Ersatzschaltbild einer chemischen Reaktionsfolge zur Analogrechnung benutzt. Die Analogiebeziehungen zwischen chemischen und elektrischen Größen wurden aus den jeweiligen, formal identischen Differentialgleichungen abgeleitet. Zur übersichtlichen Demonstration der angewendeten Methode wird hier von einem vereinfachten Ersatzschaltbild (Abb. 3) ausgegangen: Dabei sind die biochemischen Triebkräfte $\mathrm{X}_{\mathrm{i}}$ summarisch durch das konstante Potential $U_{G}$ der Gleichstromquelle am Eingang des Vierpols abgebildet. Die

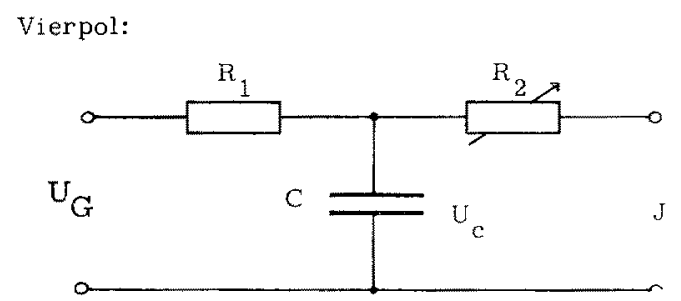

Registrierkurve Typ 3

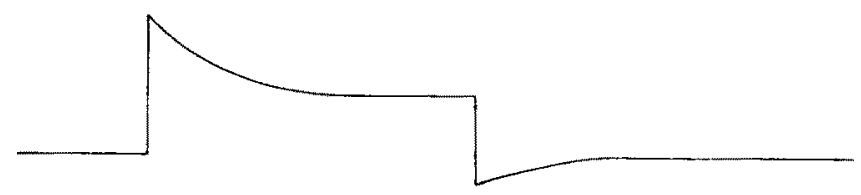

Elektrische Transportgleichungen:

$$
\begin{gathered}
J_{1}=\frac{U_{B}}{R_{11}+R_{21}} \quad J_{2}=\frac{U_{C}}{R_{22}} \quad J_{\text {st }}=\frac{U_{B}}{R_{12}+R_{22}} \\
\left(R_{11} \text { bezw. } R_{21}=\right.\text { Widerstandswerte bei "tiefer Temperatur" } \\
R_{12} \text { bezw. } R_{22}=\text { Widerstandswerte bei "höherer Temperatur" } \\
\left.U_{B}=\text { konst. }\right)
\end{gathered}
$$

Abb. 3: Reproduktion des Leistungskurvenverlaufes des Adaptationstyps 3 am reduzierten Ersatzschaltbild 
Geschwindigkeitskoeffizienten $L_{\mathrm{ik}}$ der Bildungs-- und Speicherungsgeschwindigkeit energiereicher Metaboliten im anabolen Stoffwechselzweig werden im steady state durch den reziproken Wert des Widerstandes $R_{1}$ und bei Fließgleichgewichtsübergängen durch den reziproken Wert der Zeitkonstanten $\mathrm{R}_{1} \mathrm{C}$ dargestellt. Die Anstaukonzentration der energiereichen Metaboliten vor der energiefreisetzenden und leistungsbestimmenden Reaktion wird durch das Kondensatorpotential $U_{C}$ abgebildet. Hierbei handelt es sich um intermediäre, d. h. variable Triebkräfte. Den Geschwindigkeitskoeffizienten der leistungsbestimmenden Reaktion entspricht der reziproke Wert des Widerstandes $\mathrm{R}_{2}$ bzw. der Zeitkonstanten $\mathrm{R}_{2} \mathrm{C}$ im katabolen Stoffwechselzweig. Zur Abbildung der bei Temperaturadaptation ausgelösten energetischen Flußgrößen wird im einfachsten Falle ein aktivierender Temperatureinfluß auf die Leistungsreaktion angenommen. Der Leitwert von $R_{2}$ ist dementsprechend zu verändern. Bei zeitlich rechteckiger Veränderung ist im Ausgangszweig des Modelles ein Stromstärkenverlauf zu registrieren, welcher der Adaptationskurve nach Typ 3 entspricht. Der Widerstand $R_{1}$ bleibt bei dieser Modellumschaltung in Analogie zur relativ temperaturunabhängigen Diffusionsgeschwindigkeit der Metaboliten zum Reaktionsort unverändert.

Zur Reproduktion der Adaptationskurven nach Typ 1 und 2 muß der Widerstand $\mathrm{R}_{1}$ gegensinnig zum Widerstand $\mathrm{R}_{2}$ verändert werden. Dieser Umschaltvorgang bildet damit biochemische Rückkoppelungsmechanismen des Stoffwechsels ab. Letztere treten nach HoLzER (1962) immer dann auf, wenn ein Reaktionspartner in einer Kette durch stöchiometrische Reaktion mit einem anderen Intermediat seine eigene Bildung fördernd oder hemmend beeinflußt (Endprodukthemmung, ATP-ADP-Kontrolle, EnzymSubstratkontrolle usw.; vgl. Referat WIESER). Zur Abbildung der Adaptationstypen 4

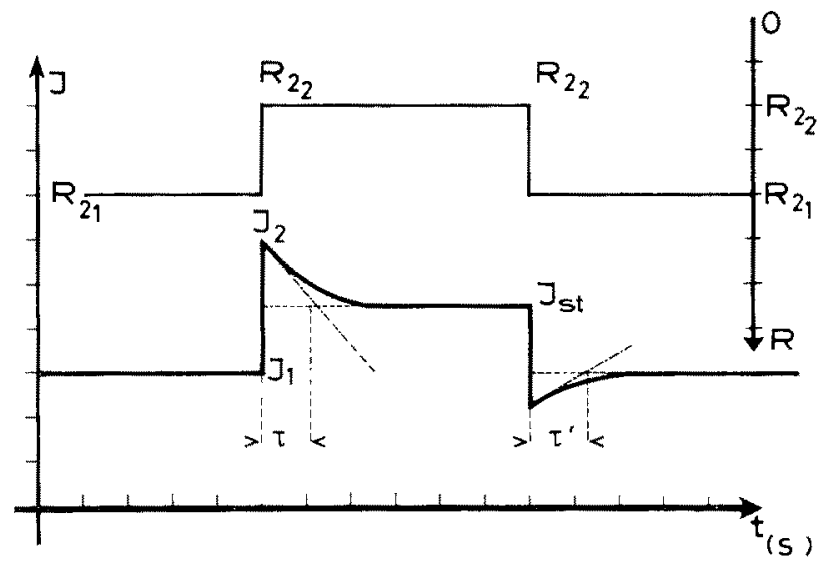

Abb. 4: Schematische Darstellung des Kurvenganges bei Adaptationstyp 3. R21 und Rog: Widerstandsgrößen des Widerstandes im Ausgangszweig des Vierpoles, $\mathrm{R}_{21}>\mathrm{R}_{22}$. J: Stromstärke, gemessen im Ausgangszweig, $J_{1}, J_{2}$ und $J_{s t}$ entsprechen den "Leistungsgrößen " $\mathrm{k}_{1}, \mathrm{k}_{2}$ und $k_{\text {st }}$ des Schemas der Abbildung 1. $\tau$ und $\tau^{\prime}$ : Zeitkonstanten der steady-state-Ubergänge von J. Die durch einfach gestrichelte Linien (-...) abgeteilten Kurvenflächen entsprechen jeweils der "cost of transition" der steady-state-Ubergänge. -....- entspricht den Subtangenten bei graphischer Bestimmung der Zeitkonstanten 
Tabelle 1

Formaler Ansatz der Gleichungen zur qualitativen und quantitativen Auswertung von Adaptationsversuchen am elektrischen Analogmodell.

(Definition der elektrischen Größen vgl. Abb. 3 und 4)

Stromstärke $\mathrm{J}_{1} \mathrm{im}$

steady state bei

"tiefer Temperatur" $T_{1}$

$$
J_{1}=\frac{U_{G}}{R_{11}+R_{21}}
$$

Stromstärke $\mathrm{J}_{2}$ sofort

nach "Temperatursprung"

von $T_{1}$ auf $T_{2}$

$J_{2}=\frac{R_{21} R_{22} U_{G}}{R_{11}+R_{21}}$

Stromstärke Jst im

steady state bei

"hoherer Temperatur" ${ }^{*} \mathrm{~T}_{2}$

$$
J_{s t}=\frac{U_{G}}{R_{12}+R_{22}}
$$

Zeitkonstante $\tau$ der steady-state-Einstellung auf $J_{\mathrm{si}}$

$$
\begin{aligned}
\tau & =\frac{\mathrm{CR}_{12} \mathrm{R}_{22}}{\mathrm{R}_{12}+\mathrm{R}_{22}} \\
\tau^{\prime} & =\frac{\mathrm{CR}_{11} \mathrm{R}_{21}}{\mathrm{R}_{11}+\mathrm{R}_{21}}
\end{aligned}
$$

Zeitkonstante $\tau^{\prime}$ der steady-state-Einstellung auf $\mathrm{J}_{1}$

"Temperaturquotient $\mathrm{Q}_{10}$ "

des Modellvorganges

bei Temperatursprung von

$\mathrm{T}_{1}$ auf $\mathrm{T}_{2}\left(\mathrm{~T}_{2}-\mathrm{T}_{1}=10^{\circ} \mathrm{C}\right)$

Relative Speicherkapazität

für potentielle Energie

$$
\mathrm{Q}_{10}=\frac{\mathrm{R}_{21}}{\mathrm{R}_{22}}=\mathrm{J}_{2} / \mathrm{J}_{1}
$$

Entropie-Erzeugung im Ausgangszweig des Vierpols

(Cost of transition)

$$
\mathrm{C}=\frac{\tau^{\prime}}{\mathrm{R}_{11} \mathrm{R}_{21}}
$$

$$
\Delta S=\frac{2}{R_{22} \tau}\left(J_{2}{ }^{2}-3 J_{S t}{ }^{2}+2 J_{2} \cdot J_{S t}\right)
$$

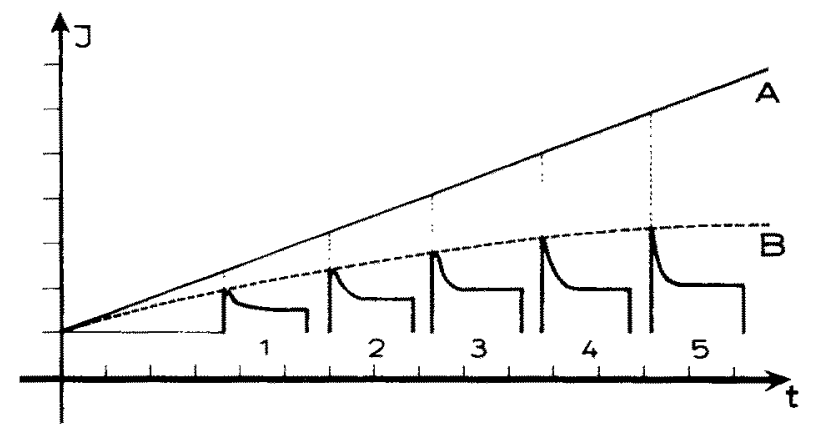

Abb. 5: Verlauf der Anpassungskurven nach Typ 3 bei stufenweiser (1, 2, 3, 4 und 5) Steigerung der "Temperatur" bzw. des Leitwertes von $R_{2}$ im Modellversuch. Abnehmende Größe der Zeitkonstanten des Einstellvorganges von 1-5. Die ausgezogene Linie A verbindet die theoretischen Spitzenwerte der "Leistungs"- bzw. Stromstärkengrơßen bei "Temperatur" - bzw. Leitwertveränderungen über die Zeit $\mathrm{O}$. Gestrichelte Linie (-...-) B verbindet die Spitzenwerte bei geringer zeitlicher Verzögerung der "Temperatur"- bzw. Leitwertänderung. 


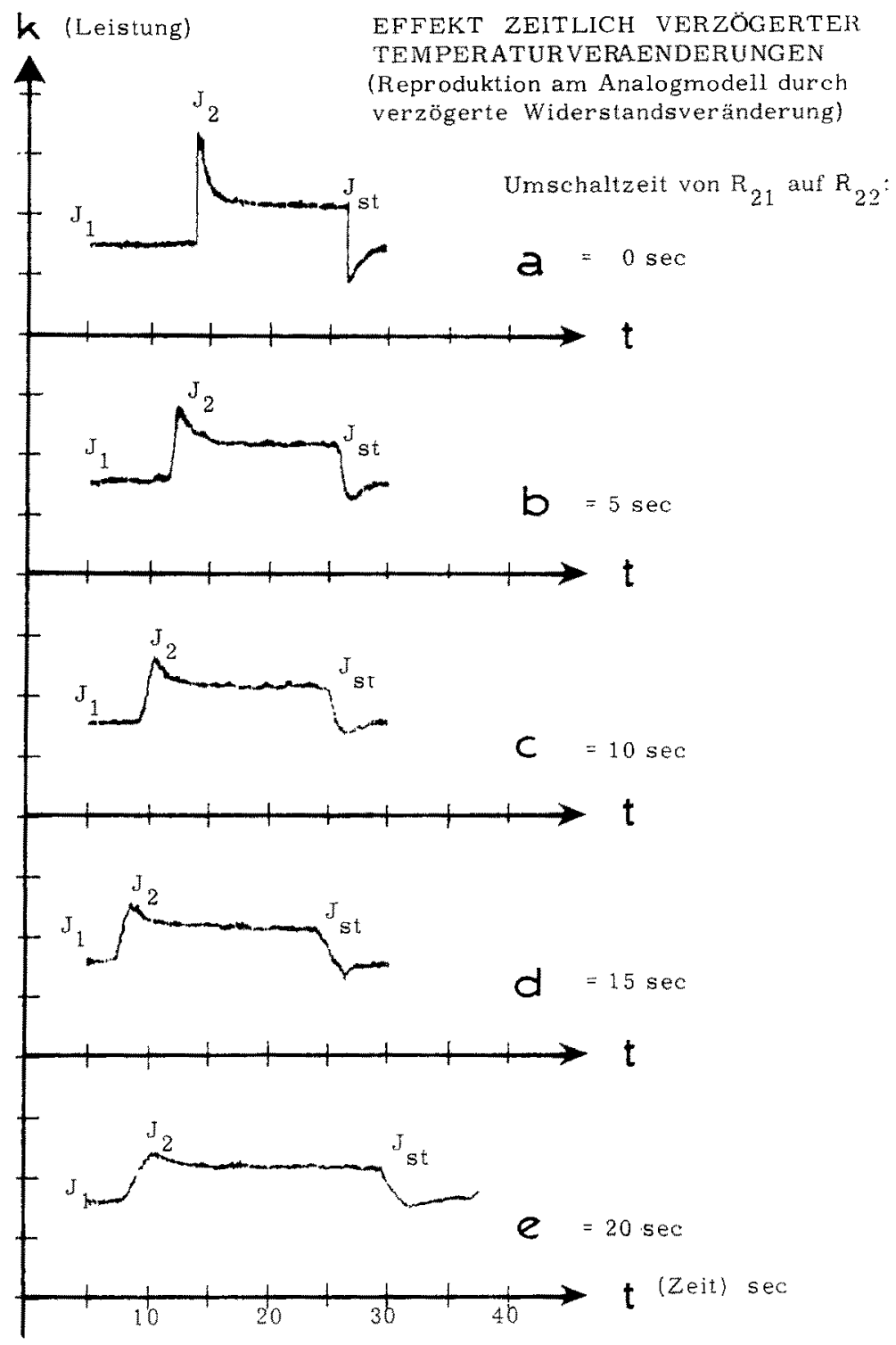

Abb. 6: Effekt zeitlich verzögerter Temperaturveränderungen

und 5 muß der Widerstand $R_{1}$ mit dem Widerstand $R_{2}$ gleichsinnig verändert werden. Auch hier sind analoge Stoff wechselvorgänge denkbar (ZERBST 1963a, b, c).

Mit diesem einfachsten Analogmodell lassen sich somit alle theoretisch möglichen Adaptationskurven phänomenologisch reproduzieren. Dabei werden nur qualitative Modellgrößen, d. h. Geschwindigkeitskoeffizienten variiert. Entsprechend den biochemischen Verhältnissen geht die Reizenergie oder die Wärmemenge nicht in den 


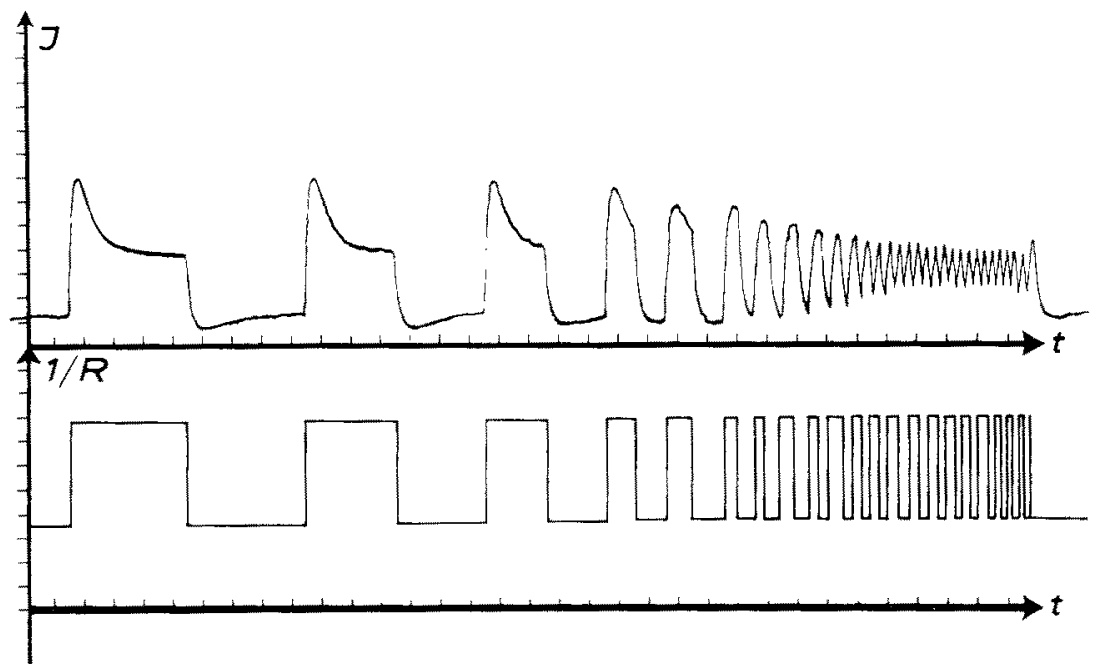

Abb. 7: Verlauf der Anpassungskurven nach Typ 3 bei abnehmender Dauer der "Temperatur" bzw. Leitwertumstellungen und zunehmender Frequenz der Umstellungen. Mit zunehmender Frequenz nehmen die Maximalwerte ron $J$ ab und die Minimalwerte $z u$. (Nachzeichnung nach einem Modellversuch)

energetischen Fluß des Systems mit ein. Alle Einzelphasen der Fließgleichgewichtseinstellungen und -übergänge sind durch - dem Ohmschen Gesetz analoge - Transportgleichungssysteme der Thermodynamik irreversibler Prozesse qualitativ und quantitativ zu beschreiben (vgl. Tab. 1). Dieses Vorgehen entspricht somit der Anwendung von Pauschalformeln, wie sie in der Chemie zur Aussage von Resultaten komplizierter, vielfach über unbekannte $Z$ wischenstufen verlaufender Reaktionen üblich sind.

Die Methode hat heuristischen Wert, wenn spezielle Vorgänge auf Grund theoretischer Ansätze simuliert und mit experimentellen Befunden verglichen werden. So haben wir z. B. am Modell den Effekt stufenweiser Temperatursteigerungen nachgeahmt. Dabei ergaben sich mit steigenden Temperaturbereichen raschere Adaptationseinstellungen (Abb. 5) auf Grund der Größenabnahme der Zeitkonstanten $\mathrm{CR}_{2}$ des steadystate-Überganges. Das stimmt mit den experimentellen Ergebnissen von BENTHE überein, der die Temperatur-Leistungsadaptation von neuromuskulären Vorgängen untersuchte. Diese Zunahme der Adaptationsgeschwindigkeit gibt auch eine Erklärung für die relative Abnahme der Temperaturquotienten $\left(\mathrm{Q}_{10}\right.$ ) biologischer Vorgänge bei höheren Temperaturbereichen. Auch einschleichende, d. h. zeitlich verzögerte Temperatursteigerungen ergaben am Modellyorgang gleiche Resultate, wie wir sie bei Untersuchungen zur Temperaturabhängigkeit der Froschherzfrequenz erhielten: Mit zunehmender Verzögerung des Temperaturganges fallen die überschießenden Leistungswerte $a b$, wie das in Abbildung 6 dargestellt ist. Bei der Nachahmung einer raschen Folge von Temperatursteigerungen und -senkungen am Modell erhält man wiederum Kurven, wie wir sie auch bei entsprechenden Experimenten am Froschherzen fanden: Mit zunehmender Temperaturwechselfrequenz kommt es zu einem graduellen Abfall der Maximalwerte und zu einem leichten Anstieg der Minimalwerte (Abb. 7), 
Nach Diskussion des Modellvorganges und seiner Auswertungsmöglichkeiten bei der Temperaturadaptation ist noch kurz auf seine Anwendungsmöglichkeit bei der theoretischen Analyse neurophysiologischer Vorgänge hinzuweisen.

Das Vierpolmodell des thermodynamisch offenen Systems ist auch als Modell biologischer Rezeptoren anzuwenden. Es zeigt alle wesentlichen Eigenschaften solcher sensorischen Meßfühler. Es verhält sich bei Reizung (Veränderung von $R_{2}$ ) wie ein PD- (Proportional-Differential-) Meßfühler, es zeigt Adaptation, Akkomodation, Summation und folgt in mittleren Reizintensitätsbereichen dem WEBER-FEchNERschen Gesetz, wenn die Reizinteusität $\left(1 / \mathbf{R}_{2}\right)$ logarithmisch gegen die Größe des Reizerfolges

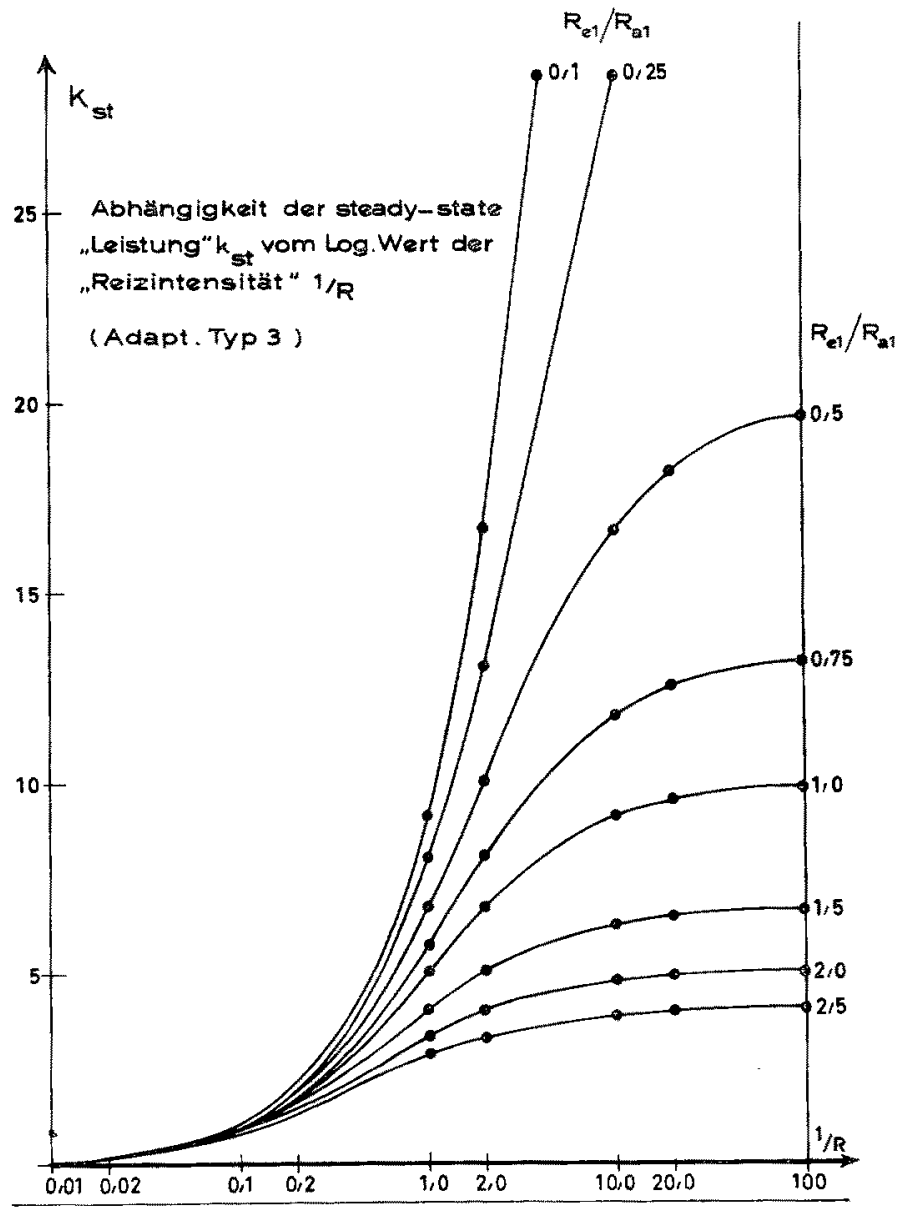

Abb. 8: Abhängigkeit des „Leistungs-steady-state“ $\mathrm{k}_{8 \mathrm{~g}}$ vom logarithmischen Wert der „Reizintensität" $1 / R_{22}$. Die Kurvenzüge verlaufen in mittleren Bereichen entsprechend dem WeberFechnerschen Gesetz näherungsweise linear. Die Kurvensteilheit ist abhängig vom jeweils gewählten Verhältnis der katabolen bzw, anabolen Umsatzgeschwindigkeit ( $\mathrm{R}_{e}$ : Widerstand im Eingangszweig des Modells, $R_{a}$ : Widerstand im Ausgangszweig). Abszissen- und Ordinatenwerte sind willkiurlich gewählte Rechenwerte. Der Kurvenzug mit dem Quotienten $R_{\mathrm{e} t} / \mathrm{R}_{\mathrm{a} 1}$ $=1,0$ entspricht dem Beispiel der Abbildung 5 
$\left(J_{s t}\right)$ aufgetragen wird (Abb. 8). Auch die Wirkung von efferenten Bahnen zum Rezeptor ist durch entsprechende Veränderungen der Widerstände abzubilden. Vorteilhafterweise wendet man hierbei Modelle an, bei denen die Widerstände durch Transistoren ersetzt sind. Reizintensitäten, hemmende oder fördernde Efferenzen können dann in Form elektrischer Spannungen angesetzt werden. Eine Registrierung von Generatorpotential und der ihm zugeordneten Impulsfrequenz von Aktionspotentialen ist simultan möglich, wenn an den Ausgangszweig des Vierpols eine Kondensator-Glimmlampenschaltung angeschlossen wird. Die Abbildung 9 gibt ein Beispiel solcher Registrierungen. Solche Rezeptor- oder Neuronenmodelle sind heuristisch wertvoll, wenn sie in Vielzahl und in entsprechend vermaschter Schaltung angewendet werden (z. B. zur Abbildung der Vorgänge bei konvergenter Neuronenschaltung der Retina des Auges). Es lassen sich dann je nach "Ableitung" die unterschiedlichsten "On-Off"-Effekte reproduzieren und analysieren. Die formale Auswertung solcher Modellversuche $\mathrm{zu}$ sinnesphysiologischen Vorgängen wird rechnerisch noch dadurch erleichtert, daß sich Größen wie „Informationsinhalt oder -kapazität" relativ einfach und dimensionsgerecht aus den elektrischen Größen als Neg-Entropie usw, bestimmen lassen.

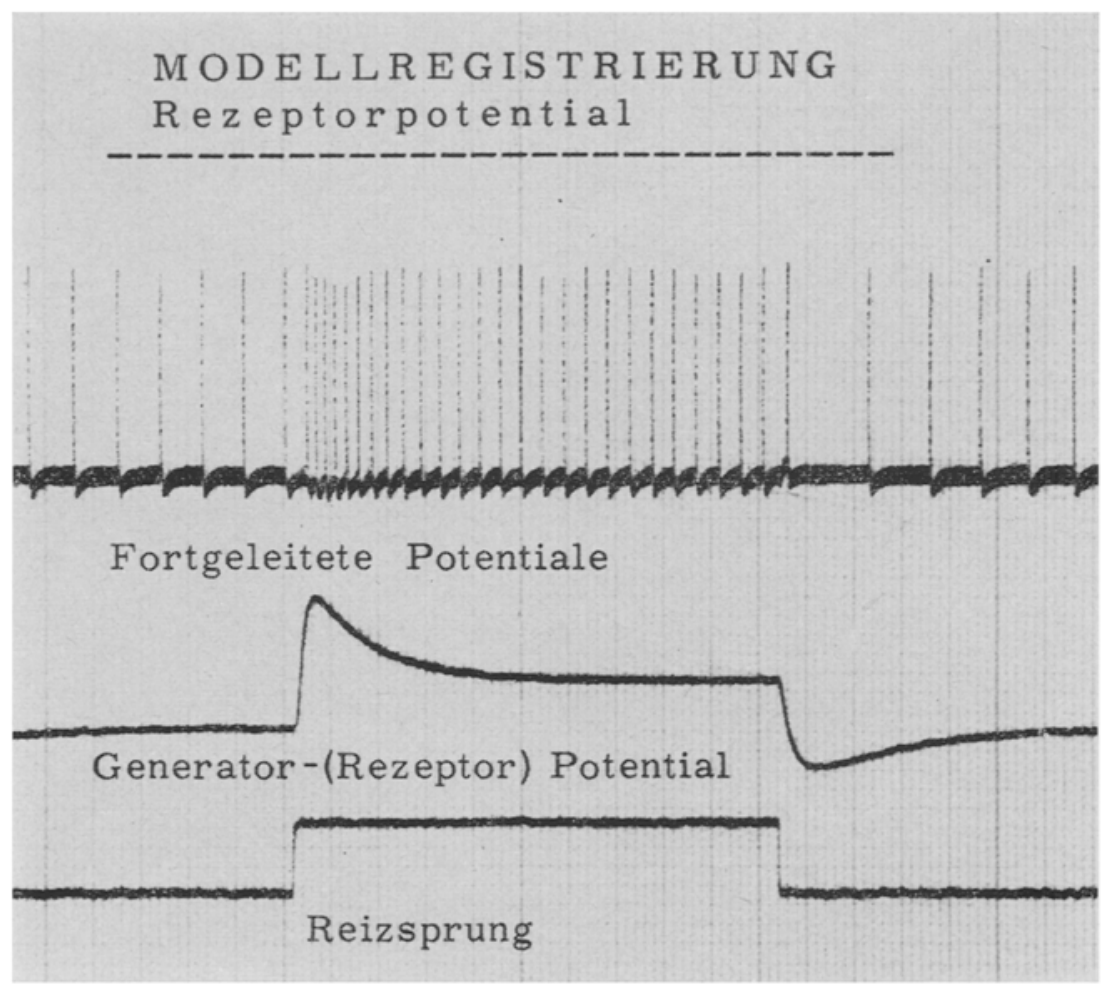

Abb. 9: Beispielregistrierung mit einem Rezeptormodell. Registrierungen von oben nach unten: Aktionspotentialfrequenz, darunter die zugeordnete Kurve des Generatorpotentials, unten Form des Reizsprunges. Das Beispiel entspricht etwa einem Ableitungsschema von On-OffSystemen von Photorezeptoren des Auges. (Originalregistrierkurve eines Modellversuches mit erweitertem Vierpol: Registrierung simultan über zwei Kanäle) 


\section{ZUSAMMENFASSUNG}

1. Biologische Anpassungsvorgänge sind aus den Grundeigenschatten des Zellstoffwechsels zu erklären. Dabei können sowohl primäre Regulationen iber Fließgleichgewichtsveränderungen und auch sekundäre Regulationen ïber Rückkoppelungsmechanismen des Stoffwechsels wirksam sein.

2. Anpassungsvorgänge sind über elektrische Analogschaltbilder von Stoffwechselzügen zu simulieren. Sie lassen sich qualitativ und quantitativ durch Transportgleichungssysteme der Thermodynamik irreversibler Prozesse beschreiben und analysieren.

3. Die Anwendung von Analogmodellen thermodynamisch offener biologischer Systeme hat heuristischen Wert für den gezielten Ansatz experimenteller Versuche an lebenden Objekten.

\section{ZITIERTE LITERATUR}

Behmann, F. W. \& Metssner, H. D., 1962. Zur nichtzentralen Temperaturanpassung des Gewebestoffwechsels. Pfingers Arch. ges. Physiol. 276, 82-88.

Benthe, F, 1954. Über die Temperaturablängigkeit neuromuskulärer Vorgänge. Z. vergl. Pbysiol. 36, 327-345.

Bertalanffy, L. v., 1951. Theoretische Biologie. Bd. 2. A. Franke, Bern, 418 pp.

- 1953. Biophysik des Fließgleichgewichts. Vieweg, Braunschweig, 60 pp.

Burton, A. C., 1939. The properties of the steady-state compared to those of equilibrium as shown in characteristic biological behavior. J. cell. comp. Pbysiol. 14, 227-349.

Densigh, K. G., Hicks, M. \& PAge, F. M., 1948. The kinetics of open reaction systems. Trans. Faraday Soc. 44, 479-495.

GRAINGER, J. N. R., 1960. The early phases in the adjustment of respiratory rates of unicellular organisms to new temperatures and the simulation of these in a model. Z. wiss. Zool. 163, $317-341$

Hafron, J. Z., 1952. Rate behavior of metabolic systems. Physiol. Rev. 32, 499-523.

Hecht, S., 1931. Die physikalische Chemie und die Physiologie des Sehakts. Ergebn. Physiol. 32, $243-390$.

Hess, B. \& CHAnCE, B., 1959. Uber zelluläre Regulationsmechanismen und ihr mathematisches Modell. Naturwissenschaften 46, 248-257.

Holzer, H., 1962. Intrazelluläre Regulation des Stoffwechsels. 102. Kongr. Dtsch. Naturforsch. 4. Arzte München.

Krebs, H. A., 1956. Die energiefreisetzenden Reaktionen des Stoffwechsels. Verh. Ber. Ges. Dtsch. Naturforsch. u. Arzte 99, 74-78.

Meixner, J. \& ReIK, H. G., 1959. Thermodynamik der irreversiblen Prozesse. In: Handbuch der Physik, Bd. III/2, Springer, Berlin, 413 pp.

Precht, H., Christophersen, J. \& Hensel, H., 1955. Temperatur und Leben. Springer, Berlin, 514 pp.

PÜTT'ER, A., 1918 bis 1920. Studien zur Theorie der Reizvorgänge. Mitteilung I-VII. Pflügers Arch. ges. Physiol. 171, 201-261; 175, 371-397; 180, 260-290.

RANKE, H., 1962. In: KeIDEL, W. D., 1962. Rankes Adaptationstheorie. Z. Biol. 115, 411-425.

ZERBsT, E., 1963a. Eine Methode zur Analyse und quantitativen Auswertung biologischer steady-state-Übergänge. Experientia, Basel 19, 166-168.

- 1963b. Die Analyse biologischer Anpassungsvorgänge mit der Methode der Thermodynamik irreversibler Prozesse. Pflügers Arch ges. Pbysiol. 277, 91.

- $1963 \mathrm{c}$. Untersuchungen zur Veränderung energetischer Fließgleichgewichte bei physiologischen Anpassungsvorgängen, Mitteilung I und II. Pflügers Arch. ges. Physiol. 277, 434-445; $446-457$. 


\section{Diskussion im Anschluß an den Vortrag ZERBST}

Grainger: I have measured the "cost of transition" in Artemia (1956; Nature, Lond, 178, 930 ) and found that it is small. In other words, the extra oxygen needed to bring the reactions

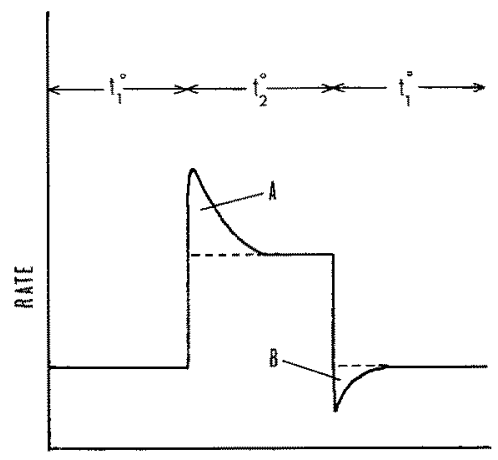

IIME

Fig. D 1: Demonstration (GRAINGER) during the discussion following the paper by ZERBST

in the animal to the new steady state levels at a higher temperature and then back again to the original steady state levels at the original temperature is small. I understood that the cost of transition is (A-B) (Fig. D 1). Is this correct?

ZERBST: Wir haben die „cost of transition" für den einmaligen Fließgleichgewichtsübergang nach Burton (1939; J. cell. comp. Physiol. 14, 335) definiert. Dazu beziehen wir uns auf das Flächenintegral des "positiven overshoot" (A). Bei der Abbildung von Receptorfunktionen ist das zur Kennzeichnung des Informationsgehaltes des PD-Meßfühlers in Form von NegEntropie besonders günstig.

PRECHT: Ich möchte auf die zur Zeit noch bestehende Schwierigkeit hinweisen, die darin liegt, den „Positiven overshoot ${ }^{\text {" }}$ von dem Adaptationsvorgang zu trennen.

ZERBst: Die Größe des overshoots ist immer abhängig von der Geschwindigkeit, mit der die Temperaturänderung vorgenommen wird. Wenn man die Temperatur einschleichend verändert, dann braucht es nicht zum overshoot zu kommen. Beim Adaptationstyp 1 ist das besonders interessant. Da können wir zu Q10-Werten unter 1 kommen. Wenn wir die Temperatur also am Modell verzögernd verringern, dann bekommen wir sofort die Tiefeinstellung und bei Erhöhung die Hocheinstellung, ohne daß es zu einem overshoot kommen müßte.

PanY: Die Möglichkeit dieser Messungen an der Retina würde wichtige Fragen der Energiebilanz im Zusammenhang mit experimentell gesetzten und an unserem Institut bisher nur mit dem ERG gemessenen retinalen Stoffwechseländerungen eröffnen.

ZERBST: Ja, das ist auch das Ziel unserer Arbeiten. Ein rein rechnerischer Ansatz hierzu findet sich bereits bei RANKE (1962; Z. Biol. 115, 411). Wir selbst haben das Analogmodell einer Receptorzelle nach zwei Gesichtspunkten entwickelt: 1. Es wird von energetischen Bilanzanalysen ausgegangen. 2. Das Modell bildet die aktiven und passiven Transportvorgänge von Kalium-, Chlor- und Natriumionen bei „Ruhe" und „Erregungsfunktionen “ ab. 